\title{
Laparostomias No Hospital João XXIII: Análise Das Indicações e Resultados
}

\author{
${ }_{1}^{1}$ Gabriela Duarte Costa Constantino, ${ }^{2}$ Domingos André Fernandes Drumond, ${ }^{3}$ Rodrigo Marques de Oliveira \\ ${ }^{4}$ Amanda Baraldi de Souza Araújo
}

\section{RESUMO}

Objetivo: Análise, através de amostragem, dos aspectos envolvidos na condução dos pacientes laparostomizados.

Materiales y métodos: Estudo prospectivo tipo série de casos. Há mais de 15 anos o serviço tem aprimorado seu protocolo em relação à laparostomia. De março/2014 a março/2016, 60 pacientes foram conduzidos em laparostomia no serviço de cirurgia geral e do trauma do Hospital Pronto Socorro João XXIII (HPSJXXIII) da Fundação Hospitalar do Estado de Minas Gerais (FHEMIG), objeto desse estudo.

Resultados: A maioria dos pacientes era jovem, média de idade de 33 anos e do sexo masculino 50 (83\%). O mecanismo de trauma mais frequente foi o penetrante 32 (53\%). O ISS maior que 16 , observado em $80 \%$ dos casos, define alto percentual de pacientes graves. A instabilidade hemodinâmica foi detectada em 39 (65\%) pacientes e 24 (40\%) foram encaminhados ao centro cirúrgico em caráter de emergência. O controle de danos foi realizado em $53(88 \%)$ das laparostomias. Ao final da primeira intervenção, a Bolsa de Bogotá foi o fechamento temporário do abdome realizado em $100 \%$ dos casos dos laparostomizados. Curativo com pressão negativa foi realizado em 13 (22\%) dos pacientes. Algum tipo de complicação abdominal ocorreu em 36 (60\%) dos casos. A fístula intestinal foi a complicação mais grave, observada em $8(13 \%)$ pacientes. O óbito foi o desfecho final em 27 (45\%) pacientes do estudo.

Conclusão: Nos últimos anos, a análise dos laparostomizados tem aguçado preocupação na condução desses pacientes no serviço. A morbimortalidade é significativa e cada vez mais há evidências de que os vieses no controle de danos acabam influenciando decisivamente nos resultados. A complacência na indicação dos procedimentos de controle de danos deve ser reavaliada nos serviços de trauma.

Relevância clínica: O crescimento do "damage control" aumentou muito a indicação de laparostomias. O abdome aberto tem benefícios bem estabelecidos no manejo de pacientes instáveis, porém, associa-se a grandes complicações e a um alto índice de morbimortalidade. É necessário maiores discussões acerca do tema e desenvolvimento de protocolos

\footnotetext{
1,4 Médica Residente do Serviço, ${ }^{2}$ Coordenador do Departamento ${ }^{3}$ Cirurgião Titular do Serviço

${ }^{1-4}$ Departamento de Cirurgia, Hospital João XXIII (FHEMIG) Belo Horizonte, Minas Gerais, Brazil

Corresponding Author: Gabriela Duarte Costa Constantino Médica Residente do Serviço, Departamento de Cirurgia Hospital João XXIII (FHEMIG), Belo Horizonte, Minas Gerais Brazil, Telefone: +5531999068123, e-mail: gabiconstantino@ yahoo.com.br
}

específicos, cada vez mais concisos, levando-se em conta os recursos humanos e materiais de cada instituição.

Palavras-chave: Abdome aberto, Controle de danos, Curativo com pressão negativa, Laparostomia.

How to cite this article: Constantino GDC, Drumond DAF, de Oliveira RM, de Souza Araújo AB. Laparostomias No Hospita João XXIII: Análise Das Indicações e Resultados. Panam J Trauma Crit Care Emerg Surg 2017;6(3):190-200.

Source of support: Nil

Conflict of interest: None

\section{ABSTRACT}

Objective: To analyse the aspects involved in the management of laparostomized patients by sampling.

Materials and methods: A prospective study of a series of cases was conducted. For more than 15 years, the service has improved its protocol regarding laparostomy. From March 2014 to March 2016, 60 patients were submitted to laparostomy in the General Surgery and Trauma's Service of the Hospital Pronto Socorro João XXIII (HPSJXXIII) of the Fundação Hospitalar do Estado de Minas Gerais (FHEMIG), as object of this study.

Results: The majority of patients (50 [83\%]) were young male and the mean age was 33 years. The predominant mechanism of trauma was penetrating $32(53 \%)$. The injury severity score (ISS) greater than 16 , observed in $80 \%$ of the cases, defines a high percentage of critical patients. Hemodynamic instability was detected in $39(65 \%)$ patients and $24(40 \%)$ were referred to the operating room as emergencies cases. The damage control method was performed in $53(88 \%)$ of the laparostomies. At the end of the first intervention, the Bolsa de Bogotá was the temporary closure of the abdomen performed in $100 \%$ of the laparostomized cases. Negative pressure wound therapy was performed in $13(22 \%)$ patients. Some kind of abdominal complication occurred in $36(60 \%)$ cases. Intestinal fistula was the most severe complication observed in $8(13 \%)$ patients. Death was the final outcome in 27 (45\%) patients in the study.

Conclusion: In the last years, the study of laparostomized patients has driven interest in the management of these patients in our service. Morbidity and mortality are significant among these cases and there is growing evidence that the damage control method and its biases ultimately influences outcomes. Compliance in the indication of damage control procedures should be reevaluated in trauma services.

Clinical significance: The growth of "damage control" has greatly increased the indication of laparostomies. The open abdomen has well-established the benefits in managing unstable patients, but it is associated with major complications

Presented in the General Research Competetion of PTS, Maceio , Brazil, November 2016. 
and a high morbidity and mortality rate. Further discussion on this topic and development of specific, increasingly concise protocols are required, taking into account the human and material resources of each institution.

Keywords: Damage control, Laparostomy, Negative pressure wound therapy, Open abdomen.

\section{INTRODUÇÃO}

Uma abordagem da parede abdominal em pacientes vítimas de trauma foi descrita por Ogilvie ${ }^{1}$ nos idos de 1940, durante a Segunda Guerra Mundial. Em 1983, Stone et $\mathrm{al}^{2}$ propuseram que abreviar a laparotomia através do tamponamento com compressas, mantendo o abdome fechado sob tensão, seria uma técnica eficiente de controlar as injúrias do trauma, a coagulopatia intraoperatória e melhorar a sobrevivência dos pacientes. Rotondo et $\mathrm{al}^{3}{ }^{3}$ em 1993, observaram que o tratamento definitivo de múltiplas lesões não tinha benefícios nos pacientes instáveis e em estado clínico crítico. O termo "controle de danos" ganhou força a partir de 1993. ${ }^{3}$ Desde então, essa tática cirúrgica tem sido usada não apenas no trauma, mas em cirurgias de emergências, em cirurgias vasculares, para controle da pressão abdominal e em quadros de sepse de foco abdominal. O objetivo é abreviar uma operação quando o paciente caminha para acidose, coagulopatia e hipotermia (tríade letal). O abdome não é fechado de forma convencional e o procedimento é interrompido após o controle do sangramento e da contaminação da cavidade. O paciente é encaminhado ao centro de tratamento intensivo e retornará ao centro cirúrgico após restabelecimento fisiológico de suas funções. ${ }^{4}$

O conceito de abdome aberto, ou seja, o não fechamento do abdome de forma convencional, define o termo laparostomia. O fechamento abdominal definitivo é conceituado como fechamento da fáscia e da pele. Deixar o abdome aberto pode aumentar significativamente o período de internação, agregar novas complicações de difícil tratamento e trazer grandes dificuldades no fechamento tardio da parede abdominal. Assim, a indicação imprecisa de laparostomia certamente agravará o estado clínico do paciente. ${ }^{4}$

O manejo do paciente laparostomizado é complexo e exige integração multiprofissional em equipe (Cirurgião, Anestesista, Intensivista, Nutrólogo, Fisioterapeuta, Enfermeiro). O paciente está sujeito à perda excessiva de líquidos, infecções, perfuração de vísceras, disfunção orgânica múltipla e morte. ${ }^{5}$ A permanência prolongada do abdome aberto pode resultar em aderências intestinais, retração da fáscia, desarranjo do domínio abdominal, formação de fistulas entéricas e desenvolvimento de grandes hérnias com difícil reconstrução da parede. .,5-7 $^{3}$ A experiência clínica tem mostrado que o manejo inicial desses pacientes define o desfecho e as complicações. ${ }^{5,8-10}$
As diferentes técnicas de fechamento abdominal alternativo e temporário dos pacientes laparostomizados vêm apresentando grande evolução ao longo dos anos. O uso de silos plásticos conhecidos como "Bolsa de Bogotá", evitando o contato direto com as alças intestinais foi inicialmente usado nesses casos. Atualmente, o uso de artifícios mecânicos (telas orgânicas e inorgânicas) usados em conjunto com a pressão negativa, parecem estar associados a avanços na reconstrução da parede abdominal. ${ }^{11-13}$

O Hospital João XXIII é um Centro de Trauma e, ao longo de anos, investiu grande esforço no entendimento e manejo do paciente crítico, nas técnicas de controle de danos e consequentemente, no paciente laparostomizado. Este estudo, apresenta dados referentes a pacientes laparostomizados, vítimas de trauma.

\section{MATERIALES Y MÉTODOS}

O estudo foi aprovado pela Comissão de Ética em Pesquisa da FHEMIG. O Termo de Consentimento Livre e Esclarecido foi usado de acordo com a Resolução do Conselho Nacional de Saúde 466/12.

Estudo prospectivo tipo série de casos que descreve e analisa dados e variáveis de pacientes submetidos a laparostomia no Hospital João XXIII. O período do estudo compreende dois anos (março/2014 a março/2016). Um total de 60 pacientes foram incluídos no estudo.

Variáveis analisadas: tempo de internação, permanência no CTI, reposição volêmica, lesões associadas, complicações e mortalidade. Os índices de trauma foram calculados de acordo com o Injury Severity Score (ISS) e o Revised Trauma Score (RTS).

Hipertensão intra-abdominal foi definida como o aumento da pressão abdominal maior ou igual a $12 \mathrm{~mm}$ $\mathrm{Hg}$. A síndrome de compartimento abdominal foi definida com a elevação da pressão intra-abdominal acima de $20 \mathrm{~mm} \mathrm{Hg}$ associado a uma nova disfunção ou falência de órgãos e/ou sistemas.

A decisão pelo controle de danos e laparostomia e, posteriormente, as técnicas de fechamento abdominal, dependem de vários fatores e varia de cirurgião para cirurgião e de instituição para instituição. Em função disto, essa variabilidade resultou em dois grupos neste estudo: (1) Aqueles com quadro clínico crítico que evoluiram com abdome aberto por período prolongado e (2) aqueles cujo abdome foi fechado com sucesso na primeira abordagem após melhora clínica com o controle de danos (geralmente nas primeiras $48 \mathrm{~h}$ ). Estes, evoluiram com menor complicação e menor taxa de mortalidade. ${ }^{5}$

\section{RESULTADOS}

No período em análise, foram admitidos no Hospital Pronto Socorro João XXIII 194.654 pacientes. Destes, 


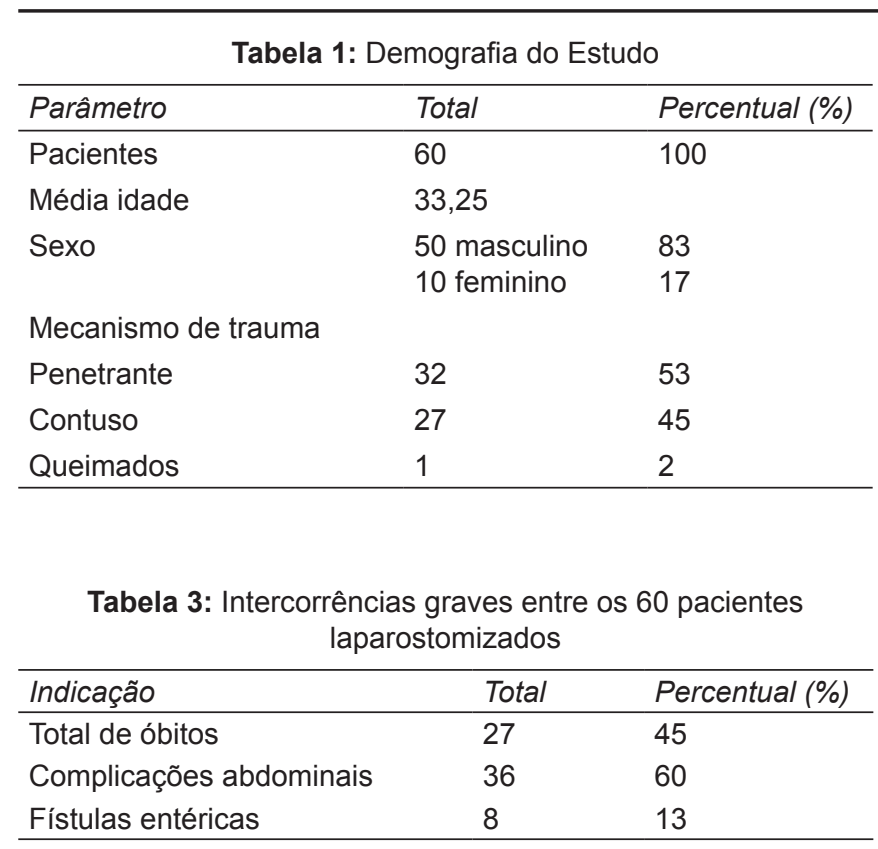

655 foram laparotomizados, dos quais 60 pacientes permaneceram em laparostomia.

A média etária foi de 33 anos e 50 pacientes eram do sexo masculino (83\%). O mecanismo de trauma mais frequente foi o penetrante 32 (53\%). O ISS maior que 16 foi observado em $80 \%$ dos casos (Tabela 1). Aproximadamente $56 \%$ dos pacientes possuíam o ISS maior que 25 à admissão hospitalar, o que define o alto percentual de pacientes graves.

A instabilidade hemodinâmica foi detectada em $39(65 \%)$ pacientes e $24(40 \%)$ foram encaminhados ao centro cirúrgico em caráter de emergência. O controle de danos foi realizado em $53(88,3 \%)$ dos laparostomizados (Tabela 2). Em 12 pacientes (20\%), o tempo cirúrgico da abordagem inicial foi superior a duas horas.

O fechamento temporário, na primeira intervenção, foi através de Bolsa de Bogotá em todos os casos. Como desfecho, 36 pacientes (60\%) evoluíram com complicação abdominal. A fístula intestinal foi a complicação mais grave e ocorreu em oito pacientes. Quatro pacientes fistulizados (50\%) estavam com a Bolsa de Bogotá. Não houve associação da ocorrência de fístulas com o uso de curativos com pressão negativa. A taxa de mortalidade dos portadores de fístula êntero-atmosférica foi de $50 \%$. O tempo de permanência hospitalar entre os que sobreviveram desta complicação variou de 40 a 194 dias.

Vinte e sete pacientes laparostomizados (45\%) faleceram nesse estudo (Tabela 3).

Trinta e três pacientes receberam alta hospitalar. $\mathrm{O}$ tempo médio entre a primeira cirurgia e o fechamento definitivo foi de 1,76 dias em 21 pacientes (fechamento junto ao tratamento definitivo das lesões). Em 7 pacientes, foi possível o fechamento através de curativo com pressão negativa. Nestes, o tempo médio entre o fechamento

\begin{tabular}{lll}
\multicolumn{3}{c}{ Tabela 2: Indicação da Laparostomia } \\
\hline Indicação & Total & Percentual (\%) \\
\hline Controle de dano & 53 & 88,3 \\
Síndrome de compartimento abdominal & 5 & 8,3 \\
Sepsis abdominal & 2 & 3,3 \\
\hline
\end{tabular}

Tabela 4: Comparação entre métodos de fechamento abdominal entre os sobreviventes

\begin{tabular}{ll}
\hline Parâmetro/métodos & Total de pacientes \\
\hline Alta com o abdome fechado & 33 \\
Fechamento na $1^{\mathrm{a}}$ reabordagem & 21 \\
- média para fechar & 1,76 dias \\
Curativo a vácuo & 7 \\
- média para fechar & 16,8 dias \\
Fechamento por $2^{\mathrm{a}}$ intenção - & 1 \\
média para fechar & 133 dias \\
Retalho cutâneo adiposo & 4 \\
- média para fechar & 76,8 dias \\
\hline
\end{tabular}

temporário e o fechamento definitivo foi de 16 dias. Em outros 4 pacientes, fez-se a opção pelo fechamento através da técnica de retalho cutâneo-adiposo, com tempo médio entre a primeira intervenção e o procedimento definitivo de 70 dias. Finalmente, em 1 paciente o fechamento ocorreu por segunda intenção. Esse paciente permaneceu hospitalizado 133 dias. (Tabela 4).

\section{DISCUSSÃO}

O trauma é uma doença de todos os povos. Conduzir um paciente, em estado crítico, vítima dessa doença, é um grande desafio. A popularidade da Cirurgia de Controle de Danos e o reconhecimento e tratamento da Síndrome de Compartimento Abdominal, além do tratamento de sepse abdominal, aumentaram muito a indicação de laparostomias. ${ }^{5} \mathrm{O}$ abdome aberto parece ter papel importante no manejo de pacientes gravemente instáveis, mas há muitas questões ainda sem respostas sobre esse assunto. A perda da arquitetura abdominal está associada a grandes complicações e morbimortalidade elevadas, além de aumentar o tempo de internação hospitalar. ${ }^{4}$

Controle de danos/laparostomia é uma estratégia de difícil decisão, sem protocolos bem estabelecidos, com indicações frequentemente subjetivas. ${ }^{14} \mathrm{O}$ sucesso do tratamento depende do desempenho da equipe cirúrgica, do envolvimento do anestesiologista e do intensivista, além da equipe paramédica. Os recursos materiais e a infra-estrutura do hospital, são decisivos na condução desses pacientes. Pacientes laparostomizados não toleram equívocos ou improvisações.

A fístula êntero-atmosférica, decorrente da exposição abdominal prolongada, é um grande desafio. O tempo de internação prolongado, a infecção abdominal persistente, a dificuldade do controle do efluente da fístula, a perda 
contínua de líquidos e a desnutrição, além dos distúrbios eletrolíticos e ácido-básicos, contribuem para os altos índices de mortalidade (acima de 40\%) associados a essa complicação. ${ }^{14,15}$

A melhor forma de se evitar esse complexo problema é a prevenção. É importante cobrir as alças intestinais, evitar a hiper-hidratação, minimizando, assim, a lesão da serosa. $\mathrm{O}$ fechamento precoce do abdome parece ser a melhor estratégia na condução desses casos. ${ }^{16}$

Neste estudo, a ocorrência de fístulas êntero-atmosféricas em oito pacientes (13\%) não teve relação direta com uso do curativo a vácuo - técnica do "Vacuum Pack" descrita por Barker e colaboradores. ${ }^{17}$ Entre os 60 pacientes laparostomizados, em apenas 13 pacientes foi aplicado o curativo com pressão negativa. Este fato decorreu da falta de recursos materiais em relação aos sistemas comerciais, como também pelo temor da aplicação do curativo com pressão negativa a Barker, visto que o sistema de aspiração negativa da instituição não permitia pressão contínua e mensuração confiável, recomendada por Barker $(100 \mathrm{~mm} \mathrm{Hg}$ a $150 \mathrm{~mm} \mathrm{Hg}){ }^{17}$

Aliado a isso, o serviço desenvolveu experiência com retalho cutâneo-adiposo para cobrir a granulação em momento apropriado. Essa técnica foi desenvolvida no serviço. Tal recurso sempre foi contemplado naqueles casos de internação prolongada, em que não foi possível fechar o abdome em curto espaço de tempo, em função das condições clínicas do paciente.

Pacientes em que o fechamento do abdome ocorreu precocemente, apresentaram melhores taxas de sobrevivência. A experiência da equipe cirúrgica e recursos materiais da instituição são decisivos para a escolha da técnica (tática) e obtenção dos melhores resultados. ${ }^{5}$

No entanto, não há estudo prospectivo randomizado controlado demonstrando superioridade de uma opção cirúrgica sobre outra, para o tratamento do abdome aberto. $^{18}$

As técnicas de fechamento abdominal passam por constante evolução e aprimoramento. O desenvolvimento delas, a menor perda de líquidos e temperatura, o uso de telas e curativos com pressão negativa foram conquistas importantes e facilitaram a condução do abdome aberto. ${ }^{11-13}$ Dessa forma, houve melhorias na sobrevida e diminuição do tempo de internação hospitalar.

Por outro lado, a mortalidade continua elevada. Nesta série, $45 \%$ dos pacientes laparostomizados faleceram.

Parece haver necessidade de agregar condutas substancialmente importantes para a manutenção desses pacientes vivos, além da estratégia de controle de danos. O caminho da hipotensão permissiva, da reposição volêmica com transfusão equilibrada de hemocomponentes e diminuição da infusão de cristalóides, são promissores nesse sentido. ${ }^{19}$ A multidisciplinaridade também precisa de absoluta sintonia, para que toda a equipe, uníssona, consiga estabelecer a sistematização do tratamento. Na experiência dos autores, a coordenação dos trabalhos e as mais críticas decisões deverão ficar a cargo de um cirurgião experiente, capaz de liderar toda a equipe e deliberar sobre o que deve ser feito em momento apropriado.

Por fim, faz-se necessário rever periodicamente os protocolos para a indicação de controle de danos / laparostomia. É importante também analisar caso a caso dos laparostomizados, além do incentivo aos estudos para uma análise crítica desse tema.

\section{CONCLUSÃO}

Nos últimos dois anos, a análise dos pacientes laparostomizados aumentou ainda mais a preocupação na condução desses pacientes no Serviço de Cirurgia Geral e do Trauma do Hospital João XXIII. A morbimortalidade é significativa e cada vez mais há evidências de que os vieses no controle de danos acabam influenciando decisivamente nos resultados. A complacência na indicação dos procedimentos de controle de danos deve ser reavaliada nos serviços de trauma. Protocolos bem definidos e equipe bem treinada podem diminuir as indicações imprecisas.

\section{RELEVÂNCIA CLÍNICA}

A popularidade do "damage control" aumentou muito a indicação de laparostomias. O abdome aberto tem benefícios bem estabelecidos no manejo de pacientes instáveis, porém, associa-se a grandes complicações e morbimortalidade elevadas. É necessário maiores discussões acerca do tema e desenvolvimento de protocolos mais concisos nos serviços.

\section{REFERÊNCIAS}

1. Olgivie WH. The late complications of abdominal war wounds. Lancet 1940;2:253-256.

2. Stone HH, Strom PR, Mullins RJ. Management of the major coagulopathy with onset during laparotomy. Ann Surg 1983 May;197(5):532-535.

3. Rotondo MF, Schwab CW, McGonigal MD, Phillips GR, Fruchterman TM, Kauder DR, Latenser BA, Angood PA. 'Damage control': an approach for improved survival in exsanguinating penetrating abdominal injury. J Trauma1993 Sep;35(3):375-382.

4. Drumond DAF. Fechamento de laparostomia com descolamento cutâneo-adiposo: uma técnica simples e eficaz para um problema complexo. Rev Col Bras Cir 2010;37(3):175-183.

5. Cheatham ML, Demetriades D, Fabian TC, Kaplan MJ, Miles WS, Schreiber MA, Holcomb JB, Bochicchio G, Sarani B, Rotondo MF. Prospective study examining clinical outcomes associated with a negative pressure wound therapy system and Barker's vacuum packing techique. World J Surg 2013 Sep;37(9):2018-2030. 
6. Cheatham ML, Safcsak K. Is the evolving management of intra-abdominal hypertension and abdominal compartment syndrome improving survival? CritCare Med 2010 Feb;38(2):402-407.

7. Miller RS, Morris JA Jr, Diaz JJ Jr, Herring MB, May AK. Complications after 344 damage-control open celiotomies. J Trauma 2005 Dec;59(6):1365-1371.

8. Acosta S, Bjarnason T, Peterson U, Pålsson B, Wanhainen A, Svensson M, Djavani K, Björck M. Multicentre prospective study of fascial closure rate after open abdomen with vacuum and mesh-mediated facial traction. Br J Surg 2011 May;98(5):735-743.

9. Björck M, Bruhin A, Cheatham M, Hinck D, Kaplan M, Manca G, Wild T, Windsor A. Classification important step to improve management of patients with an open abdomen. World J Surg 2009 June;33(6):1154-1157.

10. Cheatham ML, Safcsak K, Sugrue M. Long-term implications of intra-abdominal hypertension and abdominal compartment syndrome: physical, mental, and financial. Am Surg 2011 Jul;7(Suppl 1):S78-S82.

11. Barker DE, Kaufman HJ, Smith LA, Ciraulo DL, Richart CL, Burns RP. Vacuum pack technique of temporary abdominal closure: a 7-year experience with 112 patients. J Trauma 2000 Feb;48(2):201-206.
12. De Waele JJ, Leppäniemi AK. Temporary abdominal closure techniques. Am Surg 2011 Jul;77(Suppl 1): S46-S50.

13. Carlson GL, Patrick H, Amim AI, McPherson G, MacLennan G, Afolabi E, Mowatt G, Campbell B. Management of the open abdomen a national study of clinical outcome and safety of negative pressure wound therapy. Ann Surg 2013 Jun;257(6):1154-1159.

14. Huang YH, Li YS. Open abdomen in trauma patients: a double-edged sword. Mil Med Res 2006 Apr;3:10.

15. Becker HP, Willms A, Schwab R. Small bowel fistulas and the open abdomen. Scand J Surg 2007;96(4):263-271.

16. Ivatury RR. Update on open abdomen management: achievements and challenges. World J Surg June 2009 Jun;33(6): 1150-1153.

17. Barker DE, Kaufman HJ, Smith LA, Ciraulo DL, Richart CL, Burns RP. Vacuum pack technique of temporary abdominal closure: a 7-year experience with 112 patients. J Trauma 2000 Feb;48(2):201-206.

18. Regner JL, Kobayashi L, Coimbra R. Surgical strategies for management of the open abdomen. World J Surg 2012 Mar;36(3):497-510.

19. Higa G, Friese R, O'Keeffe T, Wynne J, Bowlby P, Ziemba M, Latifi R, Kulvatunyou N, Rhee P. Damage control laparotomy: a vital tool once overused. J Trauma 2010 Jul;69(1):53-59. 


\section{INVITADO COMENTARIO}

\section{Laparostomias No Hospital João XXIII: Análise Das Indicações e Resultados}

Incialmente gostaria de felicitar os autores por este excelente trabalho e por manter a chama da pesquisa científica viva em seu país de origem. Este e um estudo prospectivo tipo série de casos que analisa todos os pacientes vítimas de trauma admitidos neste grande centro de trauma em dois anos consecutivos, submetidos à cirurgia de controle de danos.

Os autores iniciam com uma interessante introdução remetendo os fatores históricos da cirurgia de controle de danos. A cirurgia de urgência e emergência (Acute Care Surgery) evoluiu desde os anos 50, seguindo principalmente as experiências do exército americano na Guerra da Coréia onde um modelo que identifica, prioriza e define a equipe de atendimento à urgência e trauma foi desenvolvido. Posteriormente na década de 1960, Baker e Freeark importaram este modelo do cenário militar para o cenário civil e desenharam o primeiro Centro de Trauma e o primeiro Banco de Sangue no Cook County Hospital, em Chicago nos Estados Unidos da América (EUA). Este sistema ainda incipiente focado num subconjunto de cirurgiões especialistas em trauma, emergencistas e especialistas em terapia intensiva com abordagem organizada para a avaliação e tratamento de pacientes críticos e vítimas de trauma configurou os novos centros de trauma naquele país, porém com mais tecnologia e evidências somadas da experiência de guerras mais recentes como a Guerra do Vietnam (1955 a 1975) e do Golfo Pérsico (1990 a 1991). Ainda, o avanço local da medicina pré-hospitalar também provindo da experiência de guerra, permitiu que este novo conceito de suporte de vida em campo diminuísse a mortalidade inicial dos pacientes vítimas de trauma reduzindo o tempo na cena garantindo consequentemente maior tempo para tratamento operatório definitivo, conforme necessário. Durante os 20 anos que se seguiram (1970 a 1990), os conceitos de atendimento ao trauma e emergências cirúrgicas se solidificaram sob a forma de um sistema sólido nos EUA e se propagaram pelo mundo nos 20 anos seguintes (1990 a 2010) impulsionados também, pelos esforços do Colégio Americano dos Cirurgiões implementando e divulgando os conceitos do Suporte Avançado de Vida no Trauma $\left(\right.$ ATLS $\left.^{\circledR}\right)$ até os dias atuais. Todo este valor histórico contribuiu para formação do cirurgião de urgência e trauma como modelo para o cenário mundial e brasileiro assim como a percepção da necessidade de diferenciação entre o cirurgião de trauma e o cirurgião geral por si só, incluindo a evolução do cirurgião de trauma para papéis combinados como o cirurgião especialista em lesões oriundas de trauma não ortopédico, o cirurgião de urgência e emergência (lesões não traumáticas que necessitam intervenção imediata), e o conhecimento profundo em cuidados críticos /especialidade em terapia intensiva cirúrgica. A multifuncionalidade técnica utilizada nos casos de trauma como abordagens e acessos vasculares, endovasculares, torácicos e/ou abdominais - seja por laparotomia ou videolaparoscopia - por exemplo são perfeitamente adaptáveis e utilizáveis na cirurgia de urgência e emergência.

Outro ponto alto da introdução deste artigo é a ênfase dada aos conceitos baseados em evidência atuais desta modalidade terapêutica aplicada aos pacientes fisiologicamente exaustos vítimas de trauma denominada controle de danos. Estes são importantes conceitos que fundamentam a utilização desta técnica e que justificam seu uso em pacientes críticos. Esta, considero ser uma leitura base imprescindível para o residente de cirurgia geral que precisa entender de forma correta as indicações da cirurgia do controle de danos afim de tomar a decisão crítica correta no momento correto.

O conceito de "controle de danos", (também conhecido como "laparotomia abreviada" ou "laparotomia estagiada") tem como objetivo a diminuição da imposição do estresse cirúrgico adicional em um momento de fragilidade fisiológica. A Reanimação para Controle Danos (RCD), e a Cirurgia do Controle de Danos (CCD) são um conjunto de ações deliberadas e preventivas de reanimação não-tradicionais e manobras cirúrgicas utilizadas para reverter o efeito grave da hemorragia, lesões maciças e choque. O principal objetivo é ganhar tempo com a cirurgia abreviada afim de permitir a reanimação e restabelecimento da fisiologia normal, antes da cirurgia e tratamento definitivo. Classicamente descrito para lesão abdominal catastrófica, o conceito CCD foi aplicado a todos os tipos de ferimentos. Atualmente são descritas técnicas de CCD para trauma torácico, ortopédico, vascular e trauma craniencefálico grave. De forma objetiva, esta é uma técnica através da qual o cirurgião diminui o tempo de intervenção operatória no paciente hemodinamicamente instável. A principal razão para isso é minimizar a hipotermia, a acidose metabólica e a coagulopatia ("a tríade letal"), que são muitas vezes comuns depois que o paciente perde rapidamente volume significativo de sangue, e ter sofrido trauma grave com grande destruição tecidual. Depois de um período pósoperatório de reanimação e estabilização em terapia intensiva, o paciente retorna à sala de cirurgia para tratamento 
cirúrgico definitivo. Embora os princípios sejam sólidos, extremo cuidado deve ser exercido afim de evitar o excesso de indicação deste conceito causando assim insultos secundários para vísceras. Mais do que isso, a cirurgia definitiva deve ser realizada assim que possível, a fim de minimizar a ativação da cascata inflamatória e as consequências da síndrome da resposta inflamatória sistêmica (SIRS) e disfunção orgânica. O conceito não é novo, a técnica de empacotamento hepático foi descrita por Pringle há mais de 100 anos. No entanto, com a incapacidade de compreender a lógica fisiológica subjacente, os resultados foram desastrosos. Ao longo do tempo, o conceito evoluiu para uma técnica mais moderna de laparotomia abreviada, estabelecimento do empacotamento intra-abdominal, controle de contaminação, e aplicação do tratamento definitivo após a restauração de parâmetros fisiológicos e de coagulação normais. A utilização de angiografia e embolização tem sido benéfica quando da falha do empacotamento intraabdominal, como por exemplo no trauma pélvico com hemorragia descontrolada ou destruição de tecidos moles. Mais recentemente, a combinação de CCD com um esquema de reanimação baseado em transfusão de hemocomponentes e administração acelerada de plasma e plaquetas, permitindo também hipotensão permissiva e limitando o volume de soluções cristalóides - Reanimação para o Controle Danos (RCD) - parece aumentar a sobrevida.

Ao analisar criticamente este manuscrito, algumas questões floresceram e portanto encaminho aos autores: 1 . O que aconteceria se o ponto de corte da ISS fosse selecionado em 25? Não seria esta população de pacientes críticos de fato em um centro de trauma? 2. Se os pacientes foram submetidos a DCS, por que em $20 \%$ dos casos o tempo de cirurgia foi superior a 2 horas? 3. Por que os autores usam a bolsa de Bogotá em $100 \%$ das cirurgias de controle de dano? Não seria uma escolha pior em comparação com a técnica de Barker, que é realmente uma alternativa barata? Os autores imaginam que a incidência de fístulas enteroatmosféricas seria menor se usando a técnica de Barker? 4. Qual é a explicação para $45 \%$ de mortalidade? Pacientes altamente graves (56\% com ISS > 25)? Cuidados préhospitalares imaturos? Seria a mortalidade diminuída com a aplicação dos conceitos de Reanimação Hemostática?

A Reanimação Hemostática (RH) é um complemento essencial para a laparotomia abreviada de Cirurgia do Controle de Danos (CCD), e deve ocorrer em paralelo. CCD é o tratamento proativo, preventivo da coagulopatia, hipotermia e acidose que se apresenta na presença da lesão crítica e choque. As prioridades da CCD incluem o seguinte:1. Hipotensão permissiva com reanimação a uma pressão que permita a perfusão adequada dos órgãos, ao invés de normotensão simplesmente, 2 . O uso precoce de hemoderivados ao invés de soluções cristalóides - muitas vezes facilitado pela aplicação e uso de um protocolo de transfusão maciça, 3. Administração de hemoderivados com uso liberal de PFC (Plasma Fresco Congelado) e plaquetas. A proporção ótima na terapia com hemoderivados ainda esta para ser definida, no entanto uma proporção que se aproxima de 1: 1: 1 foi sugerida. 4. Reversão de coagulopatia - TEG (tromboelastografia) ou ROTEM ${ }^{\circledR}$, se disponível, pode ser usado para orientar a terapia com hemoderivado, 5. Restauração de temperatura corpórea normal.

Em análise critica, seria importante que os autores especificassem os critérios de seleção dos pacientes admitidos para a cirurgia do controle de danos. A seleção adequada do paciente é fundamental para otimizar os resultados após a reanimação hemostática e cirurgia de controle de danos. Todos os pacientes devem ser submetidos a avaliação rápida na sala de emergência. O estabelecimento de acesso venoso de grande calibre, avaliação neurológica inicial e transfusão de sangue adequado são importantes primeiros passos assim como o diagnóstico da fonte do sangramento. A duração desta etapa é ditada pela estabilidade fisiológica do paciente, bem como a patologia subjacente. Durante esta fase, o início de um protocolo de transfusão maciça e início de uma reanimação hemostática ou reanimação para o controle de danos são críticos. Atrasos para obtenção de sala de cirurgia devem ser evitados. Manobras rápidas para controlar o sangramento externo (torniquetes, controle digital de sangramento, etc.,) são indicados enquanto ocorre a transição para a sala de operação. Em qualquer hospital que recebe vítimas de trauma ou um elevado número de casos cirúrgicos de emergência protocolos de transfusão maciça apoiando a reanimação hemostática deve estar em utilização e apoiado pela anestesia, banco de sangue e equipe de terapia intensiva. É aconselhável a solicitação de um cirurgião adicional para ajudar, esteja disponível.

As indicações para selecionar um paciente para o modo de controle de danos podem ser geralmente divididos nos seguintes: A) Instabilidade hemodinâmica (pressão arterial sistólica $<90 \mathrm{mmHg}$ durante mais que 60 minutos), B) Temperatura $<35^{\circ} \mathrm{C}, \mathrm{C}$ ) Instabilidade metabólica ( $\mathrm{pH}<7,2$; excesso de bases $>-5$; e lactato $>5 \mathrm{mmol} / \mathrm{L}$ ), D) Coagulopatia (tempo de protrombina $(\mathrm{TP})>16$ segundos, tempo de tromboplastina parcial (PTT) $>60$ segundos, E) Anatomia cirúrgica: lesão venosa maior inacessível, (e.g. veia cava retrohepática, pelve, etc.); prever a necessidade de um procedimento cirúrgico demorado em um paciente com uma resposta sub-ótima à reanimação, incapacidade de executar o reparo definitivo em tempo hábil, demanda por controle não-operatório de outras lesões, por exemplo, fratura pélvica, incapacidade para aproximar a incisão abdominal ou fechar a cavidade abdominal; F) Ambiente e Logística (necessidade de sangue $>10 \mathrm{UN}$, disponibilidade de sala de cirurgia, recursos). 
Os autores demonstram ainda importantes resultados no que concerne o fechamento ou síntese do abdome, tema em constante discussão e pesquisa na atualidade. Retorno à pergunta sobre o uso da bolsa de Bogotá como primeira escolha ( $60 \%$ com complicação, reportada no estudo) ao invés da técnica de Barker. Neste estudo aproximadamente $50 \%$ receberam alta hospitalar com o abdome fechado, contudo não está claro se a parede abdominal foi completamente fechada incluindo a aponeurose.

Do ponto de vista conceitual, a cavidade abdominal deve ser fechada afim de evitar perdas de calor e umidade, e para proteger as vísceras. Fechamento temporário é necessário quando qualquer combinação dos fatores listados acima sob controle de danos, ou nova intervenção ("second look"), existem. Em particular, pacientes com múltiplas lesões que tenham sido submetidos a cirurgia prolongada com enorme volume de ressuscitação para manter a estabilidade hemodinâmica irão desenvolver edema tissular e intersticial. Tal fato pode predispor ao desenvolvimento da síndrome compartimental abdominal (SCA) - que neste estudo somou 8.3\% dos pacientes - ou pode simplesmente fazer o fechamento primário da aponeurose impraticável. Além disso, significativa contaminação entérica ou gastrointestinal irão aumentar o risco de sepse intra-abdominal, ou a extensão do dano tecidual pode levantar dúvidas sobre a viabilidade de qualquer reparação; essas condições geralmente demandam planejamento para nova exploração e, consequentemente, fechamento temporário. Nestas circunstâncias, o fechamento abdominal temporário é necessário. O fechamento com terapia de pressão negativa (TPN) ou sub-atmosférica também mencionado pelos autores é atualmente recomendado para o tratamento do abdome aberto. Uma revisão da literatura sugere que a TPN pode ter melhorado as taxas de fechamento de cavidade, e pode estar associada com melhores resultados em relação às técnicas de fechamento abdominal alternativos (com tela, velcro, do tipo bogotá), além de diminuir a incidência de síndrome compartimental abdominal. Vale ressaltar neste ponto que a SCA pode estar presente mesmo em pacientes com abdome aberto em uso de bolsa de Bogotá, neste relato de casos em pouco menos de $10 \%$ dos casos. Merece ênfase que a "bolsa de Bogotá" e fechamento com pinça de Backaus não são mais recomendados na literatura atual.

Uma vez que o paciente tenha realizado a cirurgia definitiva e não há mais novas intervenções programadas, a parede abdominal deve ser fechada. Na maior parte dos estudos os pacientes podem ter a aponeurose fechada após a primeira re-intervenção, ou no prazo de sete a oito dias após o trauma, dependendo do tamanho e hábitos de vida do paciente, tempo para alcançar a estabilização fisiológica, volume de fluido usado para reanimar e lesões encontradas, como demonstrado pelos autores deste manuscrito que demonstraram que 21 pacientes de 33 obtiveram sucesso no fechamento da aponeurose na primeira re-intervenção.

Fechamento programado será necessário uma vez que as razões para a cirurgia temporária tenham sido tratadas. Isso geralmente é possível após um intervalo de 24 - 48 horas (ou mais). Normalmente, a parede abdominal pode ser fechada em camadas usando técnicas de síntese normais. No entanto, isto só deve ser realizada se não há nova cirurgia programada, e deve ser realizado sem qualquer tensão sobre a fáscia. Se as vísceras abdominais se mostram salientes acima da aponeurose em posição supina no paciente anestesiado, recomenda-se maior planejamento no fechamento temporário utilizando inclusive dispositivos adjuvantes no auxílio da síntese abdominal (Terapia de Pressão Negativa). Nestes casos retorne à sala de cirurgia depois de aproximadamente 24 - 48 horas de cuidados intensivos e balanço hídrico negativo ou igual a zero para então, permitir o fechamento da aponeurose abdominal. Ambos, cirurgião e anestesista devem estar cientes do perigo de causar SCA como resultado do fechamento da cavidade, e as pressões intra-abdominais ou respiratórias devem ser acompanhados de perto.

Se, por várias razões, o fechamento primário em fase tardia não for possível, então será necessário aceitar (porém minimizar) o defeito de parede resultante. Normalmente nestes casos a pele e aponeurose da parede abdominal retraem lateralmente, e, assim, impedem a síntese primária de ser alcançada. Durante o período de abdome aberto, várias técnicas são geralmente combinadas para chegar a um ponto em que o abdome anterior possa ser fechado sem tensão e com segurança. O objetivo é conseguir o fechamento aponeurótico, e subsequentemente o fechamento da pele.

Por fim, este artigo demonstra a experiência de um serviço de ponta no Estado de Minas Gerais, Brasil e que possui destaque dos profissionais que ali trabalham oriundo da dedicação e amor com que exercem seu trabalho há anos. Parabenizo uma vez mais os autores.

Bruno M Pereira

President - World Society of the Abdominal Compartment, WSACS

Director - Brazilian Society of Trauma, Chapter São Paulo, SBAIT

Associate Professor of Surgery \& Surgical Critical Care - University of Campinas, São Paulo, Brazil 


\section{INVITED COMMENTARY}

\section{Laparostomies in João Xxiii Hospital: Analysis of Indications and Results}

First, I would like to congratulate the authors for this excellent work and for keeping the flame of scientific research alive in their country of origin. This is a prospective, case-series study that examines all trauma victims undergoing damage control surgery (DCS) admitted to a major trauma center in two consecutive years.

The authors begin with an interesting introduction referring to the historical factors of the surgery of damage control. Acute care surgery has evolved since the 1950s, following mainly the experiences of the US Army in the Korean War where a model that identifies, prioritizes, and defines the emergency and trauma care team was developed. Later in the 1960s, Baker and Freeark imported this model from the military to the civilian setting and designed the first Trauma Center and the first Blood Bank at Cook County Hospital in Chicago, United States. This still incipient system focused on a subset of trauma surgeons, emergency and intensive care specialists with an organized approach to the evaluation and treatment of critical patients and victims of trauma. This led to new trauma centers in that country, but with more technology and evidence added from the experience of more recent wars such as the Vietnam War (1955-1975) and the Persian Gulf War (1990-1991). Moreover, the local advances of prehospital medicine, also derived from the experience of war, allowed this new concept of life support in the field to reduce the initial mortality of trauma patients by reducing the time on the scene, thus guaranteeing more time for definitive operative treatment, as necessary. During the next 20 years (1970 to 1990), these concepts of trauma care and surgical emergencies solidified and thus spread throughout the world in a further 20 years (1990 to 2010) by the efforts of the American College of Surgeons and its Advanced Trauma Life Support (ATLS®) to the present day. All of this contributed to the formation of the emergency and trauma surgeon as a model for the world, including Brazil. The need for differentiation between the trauma surgeon and the general surgeon was perceived. Trauma surgeons' role evolved as the specialist surgeon in injuries, whether nonorthopedic, nontraumatic injuries requiring immediate intervention, with a deep knowledge in critical care/specialty in intensive surgical therapy. The multifunctional techniques used in trauma cases such as vascular, endovascular, thoracic, and/or abdominal approaches and accesses, either by laparotomy or video laparoscopy for example, are perfectly adaptable and usable in emergency surgery.

Another high point of this article is the emphasis given to the current evidence-based concepts of this therapeutic modality applied to the physiologically exhausted patients called damage control. These are important concepts that support the use of this technique and justify its use in critically ill patients. This, I consider, is basic reading essential for the general surgery residents who need to correctly understand the indications of DCS in order to make the correct critical decision at the correct time.

Damage control surgery (also known as laparotomy or staged laparotomy) aims to reduce the imposition of additional surgical stress at a time of physiological fragility. It is a set of deliberate and preventive nontraditional resuscitation actions and surgical maneuvers used to reverse severe bleeding, massive injury, and shock effects. The main objective is to gain time with the abbreviated surgery in order to allow the resuscitation and reestablishment of normal physiology before surgery and definitive treatment. Classically described for catastrophic abdominal injury, the DCS concept is now applied to all types of injuries. Currently, DCS techniques are described for thoracic, orthopedic, vascular trauma, and severe craniocephalic trauma. Objectively, this is a technique by which the surgeon decreases the time of operative intervention in the hemodynamically unstable patient. The main reason for this is to minimize hypothermia, metabolic acidosis, and coagulopathy ("the lethal triad"), which are often common after the patient rapidly loses significant blood volume, and have suffered severe trauma with great tissue destruction. After a postoperative period of resuscitation and stabilization in intensive care, the patient returns to the operating room for definitive surgical treatment. Although the principles are sound, extreme care should be exercised in order to avoid overuse of this concept, thereby causing secondary insults to viscera. Moreover, definitive surgery should be performed as soon as possible in order to minimize the activation of the inflammatory cascade and the consequences of systemic inflammatory response syndrome and organ dysfunction. The concept is not new; the hepatic packaging technique has been described by Pringle for more than 100 years. However, with the inability to understand the underlying physiological logic, the results were disastrous. Over time, the concept evolved to a more modern technique of abbreviated laparotomy, establishment of intra-abdominal packaging, control of contamination, and application of definitive treatment after restoration of normal physiological and coagulation parameters. The use of angiography and embolization has been beneficial during failure of intra-abdominal packaging, such as in pelvic 
trauma with uncontrolled hemorrhage or destruction of soft tissues. More recently, the combination of DCS with a blood-transfusion-based resuscitation scheme and accelerated plasma and platelet administration, also allowing permissive hypotension and limiting the volume of crystalloid solutions-hemostatic resuscitation (HR) for damage control-appears to increase survival.

In critically examining this manuscript, I would pose these questions for the authors: (1) What would happen if the ISS cut-off point was selected at 25 ? Would not this critical patient population actually be in a trauma center? (2) If the patients were submitted to DCS, why was the time of surgery superior to 2 hours in $20 \%$ of the cases? (3) Why do authors use the Bogota bag in 100\% of damage control surgeries? Would it not be a worse choice compared with the Barker technique, which is really a cheap alternative? Do the authors imagine that the incidence of enteroatmospheric fistulas would be lower if using the Barker technique? (4) What is the explanation for $45 \%$ mortality? Highly severe patients (56\% with ISS > 25)? Immature prehospital care? Is mortality reduced with the application of the concepts of HR, also called damage control resuscitation (DCR)?

Damage control resuscitation is an essential complement to the abbreviated laparotomy of DCS, and should occur in parallel. Damage control surgery is the proactive, preventive treatment of coagulopathy, hypothermia, and acidosis that presents as a result of critical injury and shock. The priorities of DCR include the following: (1) permissive hypotension with resuscitation at a pressure that allows adequate perfusion of the organs, rather than simply normotension; (2) early use of blood products rather than crystalloid solutions-often facilitated by the application and use of a massive transfusion protocol; (3) hemostatic, liberal use of frozen fresh plasma and platelets. The optimal ratio in blood product therapy is yet to be defined; however, a ratio approaching 1:1:1 has been suggested; (4) reversal of coagulopathy - thromboelastography or ROTEM®, if available, can be used to guide therapy with blood derivatives; (5) restoration of normal body temperature.

In critical analysis, it would be important for the authors to specify the criteria for selection of patients admitted for DCS. Proper patient selection is critical to optimize outcomes after DCS and DCR. All patients should undergo rapid assessment in the emergency room. Establishing large-caliber venous access, initial neurological assessment, and adequate blood transfusion is an important first step as well as diagnosis of the source of bleeding. The duration of this step is dictated by the patient's physiological stability as well as the underlying pathology. During this phase, initiation of a massive transfusion protocol and initiation of a hemostatic resuscitation or resuscitation for damage control are critical. Delays to obtain operating room should be avoided. Rapid maneuvers to control external bleeding (tourniquets, digital bleed control, etc.) are indicated while the transition to the operating room is taking place. In any hospital that receives trauma victims or a high number of emergency surgical cases massive transfusion protocols supporting hemostatic resuscitation must be in use and supported by anesthesia, blood bank, and intensive care team. It is advisable to request an additional surgeon to assist, if available.

Hemodynamic instability (systolic blood pressure $<90 \mathrm{~mm} \mathrm{Hg}$ for more than 60 minutes), temperature $<35^{\circ} \mathrm{C}$, metabolic instability (prothrombin time, PT) $>16$ seconds, partial thromboplastin time $>60$ seconds, coagulopathy (PT) > 16 seconds, surgical anatomy: greater inaccessible venous lesion (e.g., retrohepatic vena cava, pelvis, etc.) anticipate the need for a delayed surgical procedure in a patient with a suboptimal response to resuscitation, inability to perform the definitive repair in a timely manner, demand for nonoperative control of other lesions, for example, pelvic fracture, inability to approach the abdominal incision or close the abdominal cavity; environment and logistics (blood need $>10$ units, operating room availability, resources).

The authors also show important results regarding the closure or synthesis of the abdomen, a topic that is constantly being discussed and researched. Return to the question about the use of the Bogota bag as the first choice (60\% with complications, reported in the study) rather than the Barker technique. In this study, approximately $50 \%$ were discharged with the abdomen closed; however, it is unclear whether the abdominal wall was completely closed including fascia.

From a conceptual point of view, the abdominal cavity must be closed in order to avoid losses of heat and moisture, and to protect the viscera. Temporary closure is required when any combination of the factors listed above under damage control, or new intervention ("second look"), exist. In particular, multiple lesion patients who have undergone prolonged surgery with massive resuscitation volume to maintain hemodynamic stability will develop tissue and interstitial edema. This may predispose to the development of abdominal compartment syndrome (ACS), which in this study accounted for $8.3 \%$ of patients, or may simply make the primary closure of the fascia impracticable. In addition, significant enteric or gastrointestinal contamination will increase the risk of intra-abdominal sepsis, or the extent of tissue damage may raise doubts about the feasibility of any repair; these conditions usually require 
planning for new exploration and, consequently, temporary closure. In these circumstances, temporary abdominal closure is necessary. Closure with negative tracheal pressure (NTP) or subatmospheric therapy also mentioned by the authors is currently recommended for treatment of the open abdomen. A review of the literature suggests that NTP may have improved closure rates, and may be associated with better results in relation to alternative abdominal closure techniques (with mesh, Velcro, Bogota bag), and to reduce the incidence of ACS. It is worth mentioning that ACS may be present even in patients with open abdomen under the Bogotá bursary, in this case report in less than $10 \%$ of the cases. It is worth emphasizing that the "Bogotá bag" and Backhaus clamp closure are no longer recommended in the current literature.

Once the patient has had the final surgery and there are no further scheduled interventions, the abdominal wall should be closed. In most studies patients may have closed the fascia after the first reintervention, or within 7 to 8 days after trauma, depending on the patient's size and living habits, time to achieve physiological stabilization, volume of fluid used to reanimate, and lesions found, as demonstrated by the authors of this manuscript, who demonstrated that 21 patients out of 33 were successful in closing the fascia in the first reintervention.

Scheduled closure will be necessary once the reasons for temporary surgery have been addressed. This is usually possible after an interval of 24 to 48 hours (or more). Normally, the abdominal wall can be closed in layers using normal synthetic techniques. However, this should only be performed if there is no further surgery scheduled, and should be performed without any tension on the fascia. If the abdominal viscera are protruding above the fascia in the supine position in the anesthetized patient, greater planning in the temporary closure is recommended, including using adjuvant devices to aid the abdominal synthesis (negative pressure therapy). In these cases return to the operating room after approximately 24 to 48 hours of intensive care and negative fluid balance allow the closure of abdominal fascia. Both surgeon and anesthetist should be aware of the danger of causing ACS as a result of cavity closure, and intra-abdominal or respiratory pressures should be monitored closely.

If, for a variety of reasons, late primary closure is not possible, then it will be necessary to accept (but minimize) the resulting wall defect. Usually in these cases the skin and fascia of the abdominal wall retract laterally, and thus prevent the primary synthesis from being achieved. During the open abdomen period, several techniques are usually combined to reach a point where the anterior abdomen can be closed safely without tension. The goal is to achieve fascia closure, and subsequently closure of the skin.

Finally, this article demonstrates the experience of a state-of-the-art service in the State of Minas Gerais, Brazil, which highlights the professionals who work there and the dedication and love with which they have been working for years. I congratulate the authors once again.

Bruno M Pereira

President, World Society of the Abdominal Compartment, WSACS

Director, Brazilian Society of Trauma, Chapter São Paulo, SBAIT Associate Professor of Surgery \& Surgical Critical Care, University of Campinas, São Paulo, Brazil 